\title{
Tangence
}

\section{L'onde amère... ment ! La souffrance dans De ma blessure atteint, et autres détresses d'André Carpentier}

\section{Olivier Aucagne}

Numéro 50, mars 1996

Lectures de nouvelles québécoises

URI : https://id.erudit.org/iderudit/025895ar

DOI : https://doi.org/10.7202/025895ar

Aller au sommaire du numéro

Éditeur(s)

Tangence

ISSN

0226-9554 (imprimé)

1710-0305 (numérique)

Découvrir la revue

Citer cet article

Aucagne, O. (1996). L'onde amère... ment ! La souffrance dans De ma blessure atteint, et autres détresses d'André Carpentier. Tangence, (50), 101-117.

https://doi.org/10.7202/025895ar d'utilisation que vous pouvez consulter en ligne.

https://apropos.erudit.org/fr/usagers/politique-dutilisation/ 


\title{
L'onde amère... ment! La souffrance dans $D e$ ma blessure atteint, et autres détresses ${ }^{1}$ d'André Carpentier
}

\author{
Olivier Aucagne
}

Le sentiment angoissant n'est lié qu'accidentellement à un objet, et il fait précisément apparaître que cet objet à cause duquel on se perd dans une mort sans terme est insignifiant pour le sentiment qu'il provoque et pour l'homme qu'il met à la torture. On meurt d'imaginer perdu n'importe quel objet de son attachement et, de cet effroi mortel qu'on ressent, on ressent aussi que cet objet n'est rien, n'est qu'un signe interchangeable, une occasion vide.

MaUrice Blanchot, Faux pas

L'onde sur une vaste étendue d'eau. Frémissante, elle agite à la surface plane du lac de minuscules oscillations; éphémère, elle s'épanouit puis disparaît sans laisser d'autres traces que son souvenir. Il est des regards qui produisent le même sentiment de mouvante éternité. Celui d'André Carpentier par exemple. Dans ses nouvelles, ses dernières productions me touchant plus particulièrement, l'écume de la douleur vient briser la quiétude du jour. Comme une légère pression des doigts sur la sérénité trompeuse de l'élément liquide. De la soudaine irruption d'un corps étranger à l'intérieur de soi, à travers les pores de la langue, est sécrétée une faiblesse particulière, sensible dans la réunion impromptue de deux embryons mélancoliques; et le lac, inquiétante profondeur de l'astre bleu, de s'arrimer singulièrement à

1 De ma blessure atteint, et autres détresses, Montréal, XYZ, coll. -L'Ère nouvelle *, 1990. Ce corpus est augmenté de: -L'enfant te ment!-, nouvelle incise dans le spécial $\times 10^{\mathrm{e}}$ anniversaire= d'XYZ. La retue de la nouvelle, Montréal, $\mathrm{n}^{\circ} 41$, printemps 1995 . Les références aux nouvelles renverront à ces éditions. 
102

"Une *, pour révéler cette "Lacune" existentielle: "Lac Une, début d'automne. La famille m'a installé ici, bien à l'écart [...]" ("L'enfant te ment!», p. 34). Lacune donc. Faiblesse du dedans à éprouver les sensations exogènes, à endosser cette peau que l'on ne reconnaît pas comme sienne: "Tout pour se sentir seul et à l'étroit dans le sac de son épiderme", dit le narrateur de "Dans l'œil rouge du couchant (p. 98). Il exprime ainsi le dérangement auquel le soumet la douleur. Parce qu'il lui est difficile de l'appréhender entièrement, d'en percevoir secrètement les dépendances. "La souffrance est à part", nous dit Henri Michaux. "Isolée, par notre faute, par notre manque à établir des rapports, d'elle à nous, d'elle à d'autres impressions "2. Dans "L'enfant te ment!", la blessure est ce qui scinde le titre en trois parties inégales et dont la signification est comme une parodie désabusée d'une véritable naissance. On ne naît qu'une fois. Toute renaissance accepte avec elle une part de sa vie abandonnée, un reliquat impur, une partie infime de la blessure, un angiome dont on aura su combattre l'existence. La démarche d'André Carpentier dans ses fictions se fonde donc sur un mouvement double: mouvement de scission, l'acte originel par lequel la blessure livre l'individu à des perceptions erronées, le conduit à se séparer d'autrui, l'isole dans sa souffrance; puis, à partir de cette mise en retrait, l'homme qui s'affaiblit, qui chute littéralement, devient cet autre qui se voit souffrir, être de douleur et de lucidité, l'être "sans visage" de Rilke. Comment passe-t-on du statut d'homme blessé à celui de bête traquée - par l'autre et par soi-même, tous deux porteurs de folie et de mort? C'est dans cet espace sombre, espace de la séparation, que la blessure nous atteint et que les nouvelles de Carpentier nous interrogent. $Y$ aura-t-il rémission ou divorce, rédemption ou déchéance pour ces êtres partagés, à l'image de leur auteur, entre pudeur et détachement?

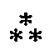

Du tréfonds de leur détresse, les personnages peints par Carpentier hésitent à se confier. Plus précisément, ils figurent la difficulté, la peine du langage balbutiant, assistant honteusement

2 Henri Michaux, «Face à ce qui se dérobe *, Paris, Gallimard, 1975, p. 7-8. 
à l'accession indécise des mots vers le néant, assorti d'un écho déformant, le son. Mieux vaudrait encore se réfugier derrière les sobriquets qui les trahissent, si peu, à l'inverse de ces baragouins inaudibles. Qu'il s'agisse de Plaisir ("Le voyage de Plaisir"), du Prince "quétaine" à la Tour abolie ("L'Ahuntsicoise"), du policier Lanoix ("Société-Pure") ou du délicat Monsieur Rosaire ("Le serment de la cuissen), dont l'existence végète dans l'ombre de ce reflet immature. Immature, parce qu'informe, et infantile (étymologiquement, l'infans est celui qui est en deça des mots ou de l'identité), que ce sobriquet arbitrairement conçu pour enfermer le grain de la voix. Donc, il faut se taire. Face à ce qui se dérobe, le silence mieux que la vacuité des discours semble assister la solitude de ces êtres au bord d'eux-mêmes. Une exception, pourtant, celle d'un certain Conteur du Cap (*Dans l'œil rouge du couchant"), dont l'excès langagier évide le langage de sa substance. Les accrocs multiples faits au verbe masquent en effet le véritable objet du discours; si bien que le bredouillement s'y transforme en bégaiement, vaine tentative d'appréhender le réel par les soubresauts involontaires de l'organe. La langue paraît ainsi condamnée à ne dire rien qui ne la serve. En sorte que cette impossibilité de dire, de se dire, qu'elle soit rentrée ou inachevée, se conjugue pour ces figures pathétiques avec la difficulté à éprouver son mal-être. Blessures indistinctes. Souvenirs enfouis sous les limbes acérées de la mémoire. L'aveu passe donc en filigrane, derrière la pudique voix d'un messager absent ou caché dans l'ombre du récit. La confession, ainsi construite, avance tel l'elfe inspiré par les creux, les vibrations du souffle contrit et souffrant; à pas comptés, elle irrigue les nervures, les brisures de la scription.

Une parole qui s'immisce ainsi, dans les saillies de l'être, entraîne le dépouillement de la pensée, l'inexprimé du saignement intérieur. À l'image de Palu, dans "Son du corps", qui "parle peu, et quand il le fait, c'est pour mieux dire son désir de silence" (p. 112). Exprimant par défaut l'effondrement du corps et l'encellement de l'esprit, il ne lui est pas permis de connaitre l'origine de sa fêlure. La souffrance demeure non assimilée. Et révéler distinctement cette incapacité, ce serait la nier puisque ce serait en être capable. Entre la parole et la blessure, s'installe donc une barrière infranchissable: la détresse, scission improductive entre le passage à l'acte et le penser. (La détresse est dans cette conscience aiguë d'une unité perdue dans laquelle l'être vit séparé de 
104

lui-même et du monde. Seul, le personnage déchiré l'est donc doublement en ce qu'il lui est impossible de communiquer à autrui son angoisse. Citons pour exemple une phrase, tirée d'un autre recueil de Carpentier, qui résume ce sentiment: "Ils voient dans le regard l'un de l'autre [...] qu'on n'est jamais parfaitement présent à soi-même en même temps que jamais présent qu'à soimême." ${ }^{3}$ ) Par cette relation en abîme, la parole devient inapte à occulter la blessure, la douleur étant antérieure à sa révélation:

Il a toujours entretenu ce sentiment très fort, devant les événements tristes, que sa tristesse était antérieure à l'événement, comme s'il avait incessamment pressenti une ancienne douleur avant qu'elle resurgît. (*Son du corps*, p. 115)

Ce primat de la blessure sur son appréhension, elle-même étant antérieure à sa divulgation, suggère l'ancrage de la souffrance dans le temps. L'émergence accidentelle d'un hématome restitue l'angoisse passée, réveille les stigmates enfouis du traumatisme originel. La persistance dans le présent d'un trouble consécutif à la répulsion, au refoulement d'une première déchirure, accentue de fait les remords silencieux qui sillonnent les souvenirs. Ainsi, l'inaptitude à vivre le présent se confond pour certains de ces personnages avec l'image obsédante d'autrefois. Dans la vieille masure où se rend Plaisir, ne persistent plus que les fragments incohérents de son enfance. Il se souvient ainsi de quelques bribes: une sœur nommée Douleur dont la perte lui fut si difficile; une mère, Aglaée, dont il n'aura connu, bien que si peu, que les frasques et les emportements. Comment vivre avec cette lancinante ignorance qui paralyse toute émotion et inflige le goût amer de l'inachevé? La réponse ne peut être que cinglante, comme une insulte à son désespoir même: *Ici s'aliène tout sentiment d'identité: le passé parle trop fort " (Le voyage de Plaisir", p. 17). Ne plus disposer d'une identité propre, cela n'est rien d'autre que disparaître sous le manteau du non-être, s'exiler un peu plus sur le chemin de l'anonymat. Or, l'anonymat fait de l'être-personnage une fissure par laquelle s'infiltre la parole de l'autre, et le condamne à se taire, à s'effacer derrière ce qui n'est pas lui. Voilà le véritable enjeu d'une telle souffrance. La douleur disperse, émiette, écrase le moi pour le livrer à la friabilité du dehors. Devant la béance de l'individu, chaque événement,

3 Carnet sur la fin possible d'un monde (*Joseph $\mathrm{K} \ldots$... inquiété par un atermoiement.), Montréal, XYZ éditeur, coll. *L'Ére nouvelle *, 1992, p. 25. 
chaque nouvelle difficulté est susceptible d'ouvrir en lui de nouvelles vannes. L'être devient poreux, perméable à la moindre intrusion de faiblesse. Ainsi, le visage même s'en trouve distendu, pareil à une baudruche dégonflée dont les rides figurent le vieillissement prématuré, jusqu'à en déformer la représentation immédiate, le reflet:

Soudain, en achevant de tourner sur lui-même pour observer la pièce dans son ensemble, Plaisir sursaute. Durant une seconde - ce qui peut paraître long dans ce genre de circonstance - il ne s'est pas reconnu dans le grand miroir au tain usé, presque déformant [...].(* Le.voyage de plaisir*, p. 19)

L'instabilité de la perception visuelle substitue de fait à l'intégrité corporelle une plasticité de la réalité physique, perçue comme un espace incertain, flou et sujet à la variabilité des tensions affectives. L'espace se modifie et se dilate dans l'expérience de la solitude. L'angoisse confère une incertitude innommable aux choses, et le personnage, se voyant privé de l'appui des autres et des repères du temps socialisé, est livré au sentiment déréalisant de sa condition misérable. (*Tout ce qui est incompréhensible ne laisse pas d'être", lit-on dans les Pensées de Pascal, au fragment 430 bis.) En conséquence, l'identité devient cette coquille vide, aspirée par le vertige de l'instant et soumise aux ballottements circonstanciels. Qu'importe alors que Plaisir apprenne par hasard sur des registres de baptême la réversibilité de ce nom supposé et porté jusqu'à ce moment-là, au profit de Douleur! Il lui semble que s'exécutè là un des tours joués par le destin, dont Rilke avait signalé la multiplicité des visages dans Les cabiers de Malte Laurids Brigge:

D'autres gens changent de visage avec une rapidité inquiétante. Ils essaient l'un après l'autre, et les usent. Il leur semble qu'ils doivent en avoir pour toujours, mais is ont à peine atteint la quarantaine que voici déjà le dernier. Cette découverte comporte, bien entendu, son tragique. ${ }^{4}$

Tout se passe donc comme si la multiplicité des visages créait chez l'être en constante rupture un défilement accéléré du temps, une parodie de sa déréliction. Appelons cela la chute libre, car celui qui s'affaisse, celui qui s'écroule littéralement sous le poids

4 Rainer Maria Rilke, Les cabiers de Malte Laurids Brigge, Paris, Seuil, coll. *Point •, traduit de l'allemand par Maurice Betz, 1966, p. 14. 
de la douleur. se perd peu à peu de vue, s'oublie dans sa chute. L'être qu'il était s'en va, et ne restent plus que des tourments définis ou une détresse indéfinie qui l'accablent. Il arrive ainsi que celui qui souffre se pose en spectateur de sa propre souffrance. Les êtres qui l'entourent, la nature qui l'isole deviennent le gigantesque miroir par lequel il entrevoit le gouffre qui l'aspire. Tel est le narrateur-personnage de "L'enfant te ment!", personnage qui, sillonnant les rues de Montréal comme on parcourt les replis de sa désespérance, c'est-à-dire pour y offrir * la caricature de sa personne, toute flétrie derrière ce regard creux, cette voix chevrotante, cette dégaine de gorille après trente ans de zoo [...]" ("L'enfant te ment!», p. 35), se trouve à devenir le spectateur involontaire et déconcerté de la chute d'un enfant (surnommé le Crapaud) depuis le balcon de l'étage supérieur d'un immeuble. Dans un élan irréfléchi, il parvient à freiner la chute de l'inconscient frondeur en faisant office de tapis de sol. L'enfant - l'enfance - lui tombe donc sur la tête. Et tout roule alors très vite dans la tête du personnage-narrateur sans nom, trop vite:

Il m'est donc tombé dessus, nul ne saurait dire par quel bout, du poids de ses cinq millions de globules rouges et quelque sept cent mètres d'intestin, de ses cent milliards de cellules de Golgi et deux cent huit os, de ses quatorze milliards de neurones corticaux et trente-cinq vertèbres, de ses cent vingt millions de bâtonnets et vingt dents temporaires, de son million d'axones pyramidaux et quelque quatre cent cinquante muscles, de ses sept millions de cônes et trois pintes de sang, de ses cinq cents centimètres cubes d'air courant et soixante pour cent d'eau. (*L'enfant te ment!*, p. 37)

L'accumulation pléthorique de détails concernant la pesanteur du "Crapaud tombeur" élargit le champ symbolique de la douleur qui n'est plus alors seulement une délitescence intérieure, masse informe s'écrasant sur l'esprit. Car la déstructuration, la dislocation de l'être qui chute signifie l'extériorisation de la souffrance, la projection hors de soi de l'image de son naufrage. Qui plus est, le ralentissement temporel de la chute agit comme un médium par lequel le lecteur que je suis - spectateur participant et interprétant - découvre la profondeur démultipliée de son abîme. Tombant avec lourdeur, mais plus encore observant un reflet singulier de ma propre dégringolade, je me dégonfle et m'affaisse, comme un pantin désarticulé, fantoche risible témoignant de son absence de structure, de sa faiblesse ontologique. 
Il y a cela dans la chute, le spectacle de cette scission intime. Une distance se creuse entre l'image projetée par la descente et l'assimilation par la conscience de ce dépouillement auquel l'individu se soumet comme en dehors de lui, dans ce qui n'est, pour Rilke, qu'une ombre "sans visage" ${ }^{5}$. Ainsi, au terme de ce processus, il ne reste donc plus à notre regard les attributs d'une personne, mais un masque, sorte de destitution de l'identité sociale, individuelle, au profit d'une neutralité bienveillante. Ce qui ne veut pas dire que l'être intime ait disparu. Seulement, il préfère s'exprimer dans l'ombre, en deçà ou à côté de lui-même, espérant sans doute, comme le Prince de "L'Ahuntsicoise", qu'une apparition sibylline viendra cautériser les plaies qui lui cimentent le coeur. Le monde n'a d'existence pour celui-ci qu'à travers sa lucarne fêlée ("Le voyage de Plaisir"), et tout semble s'y organiser de manière à restituer une cartographie mentale extrêmement précise:

Quelle que soit la position dans laquelle on s'établisse, je vous le dis, on se retrouve toujours au centre de quelque chose; chaque être s'agite à l'axe clu monde, axe tracé par lui et qu'il fait passer par le sien propre, comme devant le microcosme de ses virtualités. Voilà qu'une certaine scène se produit et les entrailles de ce monde commencent à s'agiter, jusqu'à n'en plus paraître tout à fait distraites; tout ce qui concerne l'être divise en lui la représentation des choses à l'infini. (*L'Ahuntsicoise", p. 27)

Cette géométrie de l'esprit épouse les traits d'un labyrinthe intérieur, simulant un réseau construit de multiples ramifications, de multiples développements virtuels et au centre duquel je est un hôte, la vision de soi relevant en effet d'un investissement du monde, où le je vibre à l'unisson des volutes, des modulations sismographiques subies par "les entrailles" de l'univers. Ainsi, la différence entre ce qui a lieu et ce qui n'a pas lieu, le réel et le virtuel, demeure-t-elle, pour le lecteur, difficile à établir. En conséquence, sa perception des choses s'en trouve déformée, changeant indéfiniment au gré des modifications qu'il constate, sujet en fait à une schizophrénie reliée à la multiplicité des perspectives s'offrant à lui. Cet espace modulable qu'est l'espace labyrinthique présente une duplication de la réalité qui met en relation l'extériorité de l'édifice humain et l'intériorité du sentiment. Pour

5 Rainer Maria Rilke, Les cabiers de Malte Laurids Brigge, op cit., p. 14. 
celui qui erre dans de tels dédales comme dans une nuit intérieure, les objets et les êtres parcourant les allées du quotidien se métamorphosent en une fourmilière énigmatique tamisée par le pli ondulant d'un voile :

Or, justement, un peu plus avant dans la nuit, surgissant d'un agglutinement d'insectes sinistrement gonflés de certitudes et de croyances, et qui glissent dans le clair-obscur de la ville comme s'ils géraient l'Occident, assurés que la planète ne tourne que par leur intercession, une silhouette familière, si distraite, si affairée! céruléenne ou perse ou turquine, selon l'avancement de la nuit $[\ldots]$ soudain déchire les ténèbres et commence de traverser la cour nord de sa meilleure démarche [...] ("L'Ahuntsicoise., p. 31)

On croirait apercevoir là des saillies ressortissant à un tropplein d'existence èt saturant l'espace de la jouissance langagière d'un narrateur qui déplie indéfiniment les segments de la phrase. Ce bouillonnement de mots imprime au récit un rythme viscéral; le texte prend les allures d'une danse macabre où les enroulements, les enchâssements de la phrase empruntent aux excès du baroque.

Or, pour Gilles Deleuze, le baroque, c'est justement le pli. (Étrange idée que celle de faire tenir une notion si complexe dans un seul mot de trois lettres.) Par ailleurs, on notera que cette particule est présente dans des syntagmes tels que simplification, complication, plisser, repli, rempli, déplier, multiplier, etc. Le pli naît donc du renversement d'une surface de papier ou d'une étoffe sur elle-même, de telle sorte que le pli sépare, scinde en deux parties distinctes une même matière, les réfléchissant l'une sur l'autre, produisant l'intériorisation d'une surface externe, laquelle devient surface interne. Pour paradoxale que cette appellation de "surface interne " paraisse, surface désignant étymologiquement la face du dessus, il reste que cette situation n'est pas choquante; le pli évoque une surface non plane, ondulée, boursouflée par les sursauts de l'âme. L'onde douloureuse apparaît ainsi comme un phénomène vibratoire qui entraîne la disparité des oscillations du moi et la dissolution de la forme par réfléchissement des plis entre eux. L'art baroque se définit ainsi, selon Deleuze, comme l'art de l'informel par excellence. De fait, l'informel n'est pas négation de la forme: il pose plutôt la forme comme pliée et n'existant que comme "paysage du mental , dans l'âme ou dans la tête, en hauteur; il comprend donc aussi les plis 
immatériels ${ }^{6}$. Il faut concevoir cette esthétique de l'informel, chez Carpentier, comme art de la représentation de l'illusion introspective, en ce que cette représentation exprime la sensation et le sentiment comme s'ils étaient à l'extérieur de l'individu. Suivons Plaisir à l'orée de son sommeil :

Il s'endort comme d'autres, pliés en deux, foncent tête première dans un mur de pierres. Par moments, on dirait qu'il pleure; en réalité, il rêve. Il rêve à la surface de son sommeil, ce qui fait que le gouffre d'en haut assombrit ses cauchemars. (*Le voyage de Plaisir", p. 12)

Le "paysage du mental" ainsi décrit propose la réalité à travers le prisme de l'inconscient. Deux niveaux s'en dégagent: la surface, c'est le bas où les actes demeurent inexpliqués, tandis que le haut est le règne du mystère, donc de l'esprit. On retrouve ainsi à plusieurs reprises ("Le voyage de Plaisir ", "Son du corps") une habitation, un espace physique à l'image de ce dualisme fondamental. Lorsque Palu se réfugie dans une maison pour échapper à la police, il finit par comprendre l'existence d'un étage supplémentaire où «il ne sait pas ce qu'il cherche, mais il se dit qu'il s'en apercevra quand il aura trouvé quelque chose qui compte" ("Son du corps", p. 126). À l'étage du haut s'inscrit donc la chambre centrale du labyrinthe, lieu mythique où doit s'effectuer la révélation de l'être, où l'élévation succède à la déchéance, accomplissant l'illumination souhaitée. Ces motifs symboliques structurent le cheminement du moi décrit par Marcel Brion:

Pour le voyageur qui pénètre dans le labyrinthe, le but est d'atteindre la chambre centrale, la crypte des mystères. Mais lorsqu'il l'a atteinte, il doit en sortir et regagner le monde extérieur, parvenir en somme à une nouvelle naissance [...]. Plus le voyage est difficile, plus les obstacles sont nombreux et ardus, plus ladepte se transforme et, au cours de cette initiation itinérante, acquiert un nouveau moi. ${ }^{7}$

Comme la quête du Graal, le voyage labyrinthique propose l'allégorie d'une destinée. Mais quel destin attend donc les personnages de Carpentier, généralement privés de passé? Connaîtrontils la réconciliation attendue avec eux-mêmes ou au contraire le

6 Gilles Deleuze, Le Pli. Leibniz et le Baroque, Paris, Les Éditions de Minuit, coll. * critique $\cdot 1988$, p. 49-50.

7 Marcel Brion, - Le thème de l'entrelac et du labyrinthe dans l'cuvre de Leonard de Vinci - Revue desthétique, vol. V, no 1, janvier-mars 1952. 
divorce est-il irrémissible et formera-t-il, comme pour Icare, la fin d'un rêve de cire, rêve brûlé trop vite aux flammes de l'envie?

Plaisir, devenu malgré lui son double, Douleur, juste au moment où il semblait détenir les clefs de son royaume d'ombre et de lumière, se sent projeté dans le tourbillon de la déformation impromptue de l'espace autour de lui :

Bientôt, la noirceur descend, le saisit, l'enveloppe. Comme le réduit qui l'enclôt. Il ne sait plus s'il gît sur le côté, sur le ventre ou sur le dos. Comme s'il flottait dans l'espace, il ne sait plus si c'est lui qui prend de l'expansion ou le cagibi qui rétrécit. Il sait seulement que quelque chose, les murs sans doute, informes, l'enserrent, l'étreignent. Il ne peut plus que souhaiter se soustraire à lui-même avant que la paroi ne rejoigne le milieu et ne l'écrase. ("Le voyage de Plaisir", p. 24-25)

Il apparaît dans cette étrange scène que le corps de Plaisir se soit soustrait aux lois de la pesanteur. Plaisir se fond dans l'espace, en même temps que cet espace s'adapte à la mutation de Plaisir; ainsi se produit une sorte de mimétisme corporel, entraînant la coïncidence de l'inconscient et de l'enfance. L'absence de repères spatio-temporels produit le retour de Douleur dans les limbes de la perception. Cette métaphore de la naissance, du passage à néant, de l'enveloppe utérine à la vie, se produit dans un mouvement de déréalisation et d'objectivation du corps: "Il se voit presque de loin s'anéantir, se fondre, s'absorber" "Le voyage de Plaisir", p. 25), et accentue la métamorphose de l'être. La métaphore signale de fait, comme pour le papillon, le détachement de l'encombrante chrysalide par le recours au verbe, plus précisément à le forme infinitive dans la mutation du sujet: *Devenir un autre" ( Le voyage de Plaisir", p. 25). Il ne s'agit pas du "Je est un autre", proféré par Rimbaud, qui implique le principe d'un je dans son devenir, mais bien la mise en acte d'un mouvement autonome, d'un événement détaché de l'être. Selon Gilles Deleuze:

L'infinitif pur est l'Aiôn, la forme vide ou la distance; il ne comporte aucune indication de moments, mais ne cesse de se diviser formellement dans la double direction simultanée du présent et de l'avenir. [...] Il met l'intériorité du langage en contact avec l'extériorité de l'être. ${ }^{8}$

C'est le passage de l'individuation à la mouvance du verbe.

8 Gilles Deleuze, Logique du sens, Paris, Les Éditions de minuit, coll. acritique 1969, p. 216. 
L'élan autonome qui désaisit l'homme de sa destinée, tout en lui permettant d'apprivoiser le mouvement de la vie, agit comme un spasme qui jaillirait du néant. Avant lui, tout semble éteint, morne, déhiscent. À cet appel soudain, l'être ne semble plus soumis aux contours de porcelaine qui l'habillaient, mais rompt cette captivité par un sursaut inattendu. Le corps exulte dans cette torsion qui sépare le sujet de lui-même. Il se trouve au point où les chemins bifurquent, où il ne lui est plus possible de revenir en arrière, en raison de son audace. Tel le narrateur de "L'enfant te ment!" qui ne se "sent revivre qu'aux carrefours, ces lieux de bifurcations, de hasards, de risques, de démesures" (p. 36). Plus loin, à l'instant de la bifurcation, au moment où le narrateur se retrouve seul et sans aide au milieu du lac, son embarcation ayant chaviré, se pose la question du devenir: où aller, quelle direction le rapprochera de sa vérité? Faut-il "songer à fuir, par un bout ou par l'autre, par la vie ou par son contraire [...]?" (“L'enfant te ment!", p. 43) La réponse est que plus singulière:

Il y a alors eu un moment indéfinissable où le cour a hésité. La conscience s'est éclatée durant que j'évaluais ma position au milieu de ce désert d'eau et que je cherchais à me calmer, alors que tout était paisible [...] Ainsi, j’ai convenu avec moi-même de me mettre sur la voie de la continuation, d'investir l'effort pour me sauver de l'emprise du lac. En réalité, je n'ai pas choisi, j’ai juste laissé l'événement répondre à sa propre... potentialité. ("L'enfant te ment!*, p. 45)

En choisissant de ne pas choisir, mais de laisser la pleine autonomie aux circonstances, le narrateur-personnage constate et subit sa destinée sans trop contribuer à définir sa pente. D'ailleurs, comment pourrait-il faire preuve d'un esprit de décision lorsque tout arrive à la fois malgré lui et avec son propre concours involontaire, dans l'intermittence du moment, dans la lézarde du réel, sur un mode si décisif qu'on dirait qu'il court vers un point de non-retour inconnu de lui, mais qui le fascine à l'excès? Les propriétés des personnages de Carpentier se rassemblent justement autour de ce manque d'assomption, manque qui voile cependant un déterminisme dont les données se condensent souvent dans la chute. C'est pourquoi le passé revient si souvent en force... Par ailleurs, la courbe du hasard, parce qu'elle ne passe jamais par les mêmes points, ne sollicite aucun principe. Un destin de type "coup de dés "entraîne le personnage dans un monde où rien n'est jamais joué d'avance. Certains, comme le narrateur de "L'enfant te ment!" ou l'inspecteur de "Société pure" 


\section{2}

finissent par se sauver ou par être préservés de leur noyade morale; d'autres, tels le bon Monsieur Rosaire ("Le serment de la cuisse"), le parricide Palu ("Son du corps") ou "le Prince "quétaine" à la Tour abolie" (L'Ahuntsicoise»), sombrent inexorablement.

À l'aune de ce constat, il reste à se pencher sur la deuxième partie de l'expression "chute libre", employée précédemment, en nous demandant si l'événement apparemment fortuit est bien un effet de liberté.

$$
\stackrel{*}{* *}
$$

Il semble a priori impossible d'affirmer ici quoi que ce soit, tant l'idée d'une fortuité est intimement liée à l'expression déroutante d'une imprévisibilité. Tout juste peut-on suggérer que l'action du hasard met les personnages de Carpentier sous les conditions d'une hypothétique liberté qui a plutôt les allures d'une disponibilité. Le coup du sort les surprend dans la mesure où l'inattendu paraît guidé par une intention sans qu'on puisse assigner au fatum une fin qui le justifie. Il y a là l'amorce d'une déclaration de principe du type "le hasard fait bien les choses "et en même temps la dénonciation de ce postulat, parce que sa nature est proprement immotivée. Il résulte de cette contradiction une étrange déformation de la réalité, entendue comme terrain des possibles, lieu de rencontre de l'être-personnage et de l'inattendu. Tout se passerait donc comme si le hasard conservait l'aura mystérieuse de la providence tout en ignorant l'arbitraire du signe divin. (On notera la récurrence de motifs religieux dans l'œeuvre de Carpentier.) Le pli du hasard, contrairement au signe divin qui suppose une dimension révélatrice, nous interpelle personnages et lecteurs - parce qu'il ne nous livre rien de plus consistant qu'une esquisse. Dans l'intervention divine, la finalité s'explique par une relation causale, tandis que l'instant subjugué par la contingence ne prétend pas démontrer l'évidence: il affirme la beauté du rapprochement spontané, la magie analogique de l'incidence déconcertante.

On observera aussi que l'intervention du hasard ne signifie pas forcément abandon ou délestage de l'être à toutes les éventuelles manifestations extérieures; l'automatisme ne saurait être le 
seul moyen d'éprouver le vertige du hasard. Par ailleurs, à s'exonérer de la responsabilité de ses actes, le personnage finirait par saborder l'intellection, comme tout rapport au réel et au langage. Bien plus intéressant est le projet de Carpentier d'engendrer des analogies conscientes mais inusitées entre différents lieux du sens. Tel est la signification même de l'équivoque - comme procédé littéraire. L'équivoque, ainsi qu'elle est pratiquée par exemple dans les jeux de langage (on aura noté la récurrence de tels jeux dans l'œuvre de Carpentier), produit une segmentation du syntagme, dont les fragments incidemment obtenus créent une seconde signifiance. Il s'agit de faire coexister le contenant et le contenu dans une nouvelle et insolite constellation imaginaire le contenant engendrant le contenu et inversement. En réinventant l'origine du mot à partir d'une fausse étymologie, l'veil exercé explore des voix insoupçonnées à partir d'un simple cocon morphologique. Que les lambeaux d'un être s'effritent et voici que sa lacune inavouable se révèle dans l'abîme d'un lac nommé Une. Veut-on déceler dans cet aveu les. signes d'une nouvelle naissance, que ce dernier s'exclame: halte, "l'enfant te ment!", parodiant ainsi la symbolique du corps s'extrayant du néant amniotique... (Les figures de la naissance sont nombreuses dans l'œuvre de Carpentier.)

Plus qu'un jeu lexical, l'attention portée à l'élément phonique du langage souligne un principe d'attirance, d'aimantation, qui fait du syntagme un objet linguistique aux multiples relations. L'expression ainsi obtenue après séparation éclaire en effet le premier terme au point que celui-ci se double d'une nouvelle signification capable de concurrencer la première. Dans l'équivoque, il y a ainsi l'écho sémantique entretenu par ces deux syntagmes tour à tour entremêlés, tour à tour détachés. Imitant les arabesques du corps, s'exprimant simultanément et confusément avec les épures de l'esprit, le jeu de langage contribue à multiplier les croisements de sens.

$$
* *
$$

L'ambiguité du dessein des personnages de Carpentier tient aussi en partie à la coexistence de multiples voies sur lesquelles les entraîne l'aiguillage imprévisible de la détresse. La souffrance est comme ce messager qui voudrait m'apprendre sur moi ce que 
114

je ne puis comprendre et qui utiliserait des moyens détournés pour me le signifier, à la manière de Séraphin Lange "De ma blessure atteint"), l'envoyé de Toucheur auprès de Gédé et de Papineau, dont le message obscur voile les véritables motifs de sa visite. Car ces motifs, ils se terrent "au-delà " des faits, on les dirait en allés, comme le messager de Toucheur au pays des Lacs (on y revient), exilés dans un ailleurs dont la simple évocation suffit à ranimer les vestiges enfouis d'une amitié inaltérable, malgré le temps, l'espace, les divergences qui séparent les êtres.

On croit pouvoir s'immiscer au creux de la blessure, avec la certitude que la douleur cessera dès lors que l'on aura cerné l'objet qui la provoque, dès lors que cet objet aura été connu de l'entourage; mais l'on se trompe, car on n'effleure ainsi qu'une peau déjà morte sur laquelle viendra s'incruster aussitôt une autre égratignure. La blessure, et cela, semble-t-il, constitue un véritable contrepoint dans l'écriture de Carpentier, est un trompe-l'œil. Ne nous égarons pas lorsque nous parcourons ces fils tendus sur la pointe des mots; ils nous regardent à travers leurs weillères souffrantes et semblent murmurer, comme le souvenir-écran: une onde amère... ment!

Observons l’intriguant détective Lanoix ("Société-Pure"). Sur la piste de l'assassin de plusieurs personnalités, toutes reliées par leur activisme politique, il tente de reconstituer dans son esprit embrumé le motif commun de ces attentats. Mais au moment où le puzzle commence à prendre forme dans sa tête, il se trouve brusquement enlevé par un groupe d'extrémistes qui lui révèlent peu à peu le but de leur croisade destinée à restaurer l'ordre au nom cle la pureté. Face à l'ultimatum posé par ses ravisseurs, qui lui proposent, puis le somment de se joindre à eux, Lanoix explose de colère, détruisant presque tout sur son passage et laissant derrière lui un enchevêtrement de corps et de sang. Mais il lui faut aller plus loin dans la démesure : il reconstruit, à partir du communiqué émis par le groupuscule, un autre communiqué, reprenant les termes du premier, mais cette fois-ci en revendiquant l'assassinat des extrémiste de droite par un mouvement gauchiste. Ce message se présente sous "forme de calligramme ressemblant au jet d'eau de La colombe poignardée et le Jet d'eau, d'Apollinaire." (Société pure", p. 94). Il y a derrière cette attitude le sentiment que l'objet lié à la souffrance n'est qu'accidentellement la source du mal-être. L'objet n'existe donc que comme représentation de celui-ci, avec pour conséquence de n'exprimer 
rien d'autre qu'un pis-aller masquant une autre façade de ce même mal. Sous l'angoisse, une seconde image vient refléter la première, infiniment la même parce que toujours dissemblable:

Son aveuglement est d'autant plus profond que le clésir, chez lui, n'est plus que parallèle, La paix ne lui reviendra pas, mais il l'ignore. Il prétend mimer le clésir qu'il na pas. Il croit enfin savoir à quoi il était cléterminé; il ne peut plus vivre avec le hasard. Il s"éloigne de ce qu'il est pour mieux le devenir, cest son idée fixe. ("Société pure", p. 95)

Le mime, expression d'une mise en scène de la personne, révoque ici l'idée d'une essence possible de la détresse. Le fait de dire son désarroi, c'est déjà avouer implicitement que celui-ci n'existe que par le langage. Le mot est donc un leurre par lequel, en trompant sa faiblesse, on s'abuse soi-même. Car feindre la douleur, c'est déjà fabuler sur ce qui n'existe pas hors de soi; donc" "raconter n'arrache rien, n'endort aucun mal" "L'enfant te ment!", p. 34)

Mais hors de ce concret du discours, il ne semble alors se trouver de place que pour la non-possession d'un vide. Faute d'un contact avec autre chose que ce rien silencieux, la pensée en est réduite à un babillage innocent, écho d'une vie dans les limbes. Lorsque Palu ("Son du corps"), à l'extrémité de sa fuite en avant, décide de mettre fin à ses jours, il paraît céder à l'appel du silence, ou plutôt se fige dans la répétition d'un même mot:

Contrat! quelque chose est en train de céder; son unité, sa continuité lui échappent. Ne lui reste plus que la tentation de suspendre ses gestes, de tout taire. Peu à peu, il devient sans mots; de fait, il n'entretient l'écho que d'un seul, dont il ne sait plus le sens, et qu'il répète à l'infini, sans émotion: "contrat, contrat, contrat... Puis il devient tel qu'il s'est autrefois si souvent imaginé: vivant, mais... comme mort, fermé à lá vie. Le sens du dernier mot lui échappe. Tout se défait, sauf ce qui n'est pas lui. La vie reste, le vivre s'est dissous. Palu n'est plus sensible qu'au son du corps. (. Son du corps *, p. 137-138)

Le mot devient ici invocation. Il perd son rôle de désignation, se fait scansion onomatopéique. Il restitue de fait le mouvement même de la vie. Sous le vernis du langage s'installe le silence de la mémoire assassine, pour faire place à l'innocence, à la pureté du corps seul. Car, en définitive, la dépossession ne concerne pas le seul langage. Elle est d'abord dépossession du monde et de soi. À travers cette suspension du temps, l'être retourne au rien 
qui l'a vu naître. Sous le murmure de la vie qui se défait, il y a le désir de s'exonérer de soi-même, de s'abandonner à une totale plasticité. Etre le verbe, l'eau courante du fleuve, la fluence du grain de sable.

Le livre, métaphore d'une vie dissolue, entretient enfin le mystère d'une pensée en lambeaux, d'un être introuvable parmi les pages calcinées laissées derrière lui par Palu. La blessure est ici, tout près, dans cette ironie qui met la vie sur un même plan que le catalogue Eaton. (Palu périt dans l'incendie de la maison paternelle; on le retrouvera plus tard avec un "catalogue Eaton... qu'il aura abandonné sous lui comme un signe exténué ", "Son du corps", p. 138). Le propre de cet acte, en rupture avec la vie et avec la raison, est de révéler que les signes sont en décalage avec les choses. Il n'y a pas adéquation du signe et de la vie, mais dénonciation du signe comme masque de la chose. Les signes sont interchangeables en ce qu'ils ne côtoient pas directement le réel. Seul l'acte désespéré de Palu permet de renouer avec la virginité d'un réel qu'il a contribué à défigurer. Et l'écriture ne parvient pas à extérioriser cette souffrance, sinon en la rendant indicible dans le bruissement intemporel d'une feuille qui se consume.

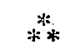

Peut-on vraiment imaginer d'épuiser la plénitude de l'échec? Peut-on même affirmer que la douleur révélée porte un message concordant avec la vérité de la fêlure? L'acte d'écrire pose, chez André Carpentier, la question de la fidélité du langage au vouloirdire. Cela met en ceuvre une quête, un cheminement du moi dont chaque nouvelle tend à se faire l'écho fragmentaire. Il ne saurait être question d'aboutir à une réponse univoque concernant cette prospection intime des vaisseaux signifiants de la pensée. Il se pourrait même qu'il n'existe d'autre réponse que l'interruption inopinée de la voix; autrement dit, que là où le grain de la voix se pose sur le blanc de l'espace, il n'y ait qu'un gribouilli indescripitible. Mais alors, si la pensée douloureuse sollicite en vain les expertises de l'écriture, ou de la parole, cela suggère que toute souffrance est chargée de la conscience de son accidentalité. Là où le hasard s'inscrit dans la chair desséchée par le sel de l'amer, il substitue à l'image du bien ou du mal l'esquisse de son 
propre reflet. Mais refléter, c'est déjà donner à voir, c'est déjà embrasser l'aporie qui nous éloigne du rivage de l'être, qui maintient dans juste assez d'eau pour garder de l'onde douloureuse le sentiment qu'elle nous glisse entre les doigts, juste assez de rien pour s'entendre dire: Voici matière à écrire! 